\title{
A VOLUME ESTIMATE FOR STRONG SUBHARMONICITY AND MAXIMUM PRINCIPLE ON COMPLETE RIEMANNIAN MANIFOLDS
}

\author{
KENSHO TAKEGOSHI
}

\begin{abstract}
A generalized maximum principle on a complete Riemannian manifold $(M, g)$ is shown under a certain volume growth condition of $(M, g)$ and its geometric applications are given.
\end{abstract}

\section{Introduction}

Let $(M, g)$ be a complete and connected Riemannian manifold of dimension $m \geq 1$. In this article we study the following problem:

Problem. For any $\varepsilon>0$ and a smooth function $f$ bounded from above on $M$ does there exist $x_{\varepsilon} \in M$ such that (i) $\sup _{M} f-\varepsilon<f\left(x_{\varepsilon}\right)$ (ii) $|\operatorname{grad} f|\left(x_{\varepsilon}\right)<\varepsilon$ and (iii) $\Delta f\left(x_{\varepsilon}\right)<\varepsilon$ ?

Omori first formulated such a maximum principle on a complete Riemannian manifold with sectional curvature bounded from below (cf. [Om]). Since then, the maximum principle has been generalized by several authors and applied to the study of value distribution of maps between manifolds as first observed by Omori, [Om]. Especially Yau generalized Omori's result to a complete Riemannian manifold with Ricci curvature bounded from below (cf. [C-Y], [Y-1]). Recently Chen \& Xin [C-X] and Ratto, Rigoli \& Setti [R-R-S] have extended the principle to a complete Riemannian manifold whose Ricci curvature decays slower than a certain decreasing function tending to minus infinity. Their curvature condition is optimal in a sense, and the idea of the proof heavily depends on an upper estimate for the Laplacian of the distance function by the curvature condition.

On the other hand it is known that the maximum principle no longer holds on a general complete non-compact Riemannian manifold and the problem is deeply related to the volume growth property of $(M, g)$. Here for any $x \in M$ and $r>0$ we define a function $h_{x}$ by $h_{x}(r):=\log V_{x}(r) / r^{2}$,

Received January 29, 1997. 
where $V_{x}(r)$ is the volume of the geodesic ball $B_{x}(r)$ centered at $x \in M$ of radius $r>0$. Every complete Riemannian manifold $(M, g)$ in the articles [Om], [C-Y], [C-X], [R-R-S], [Y-1] admits a point $x \in M$ with $\int_{1}^{+\infty} d r / r h_{x}(r)=+\infty$ in view of their curvature condition. Furthermore for a given smooth monotone increasing function $h(r)$ satisfying $\int_{1}^{+\infty} d r / r h(r)$ $<+\infty$, we can construct a two dimensional complete Riemannian manifold $\left(N, g_{h}\right)$ admitting a bounded strongly subharmonic function $u \geq 0$; i.e., $\Delta u \geq c>0$ on $N$ and $\sup _{N} u<+\infty$, and a point $x$ such that $h_{x}(r) \sim h(r)$ for sufficiently large $r$ (cf. $\S 2$, Remark 2.4). From this observation we would like to propose the following conjecture:

CONJECTURE. Suppose a complete Riemannian manifold $(M, g)$ of dimension $m \geq 1$ admits a point $x \in M$ such that $\int_{1}^{+\infty} d r / r h_{x}(r)=+\infty$. Then the maximum principle holds on $(M, g)$.

In this article we show the following theorem which is weaker than the above conjecture.

THEOREM. Let $(M, g)$ be a complete Riemannian manifold of dimension $m \geq 1$. Suppose $M$ admits a point $x$ such that $\liminf _{r \rightarrow+\infty} h_{x}(r)<+\infty$. Then the maximum principle holds on $(M, g)$.

As far as we know, this result is the first one for the maximum principle on complete Riemannian manifolds without any curvature condition and it generalizes the maximum principle obtained in $[\mathrm{C}-\mathrm{Y}],[\mathrm{Om}],[\mathrm{Kr}],[\mathrm{Y}-1]$. Our method is based on a volume estimate for strong subharmonicity on complete Riemannian manifolds. Furthermore there exists a two dimensional complete Riemannian manifold with finite volume whose Gaussian curvature decays faster than the curvature condition assumed by [C-X] or [R-R-S] (cf. [L-S], §3, Example 1).

The author would like to express his thanks to Prof. A. Atsuji during the development of this article.

\section{$\S 1$. A volume estimate for strong subharmonicity on complete Riemannian manifolds}

Let $(M, g)$ be a complete non-compact Riemannian manifold of dimension $m \geq 1$ and let $V_{x}(r)$ denote the volume of the geodesic ball $B_{x}(r)$ 
centered at $x \in M$ of radius $r>0$. Let $u$ (resp. $K$ ) be a smooth (resp. nonnegative continuous) function on $M$. We define subsets of $M$ as follows: for given constants $\alpha \geq-1, \beta>0$, we set

$$
\Omega(u, \alpha, \beta):=\left\{x \in M ; u(x) \geq 0 \text { and } \Delta u(x) \geq \beta K(x) u(x)^{\alpha+1}\right\}
$$

where $\Delta u:=\operatorname{Trace}_{g} \nabla^{2} u, \quad$ and for a given constant $\gamma>0$, we set

$$
M(u, \gamma):=\{x \in M ; u(x)>\gamma\}
$$

The following theorem plays a crucial role in this article.

THEOREM 1.1. Suppose $M(u, \gamma)$ is a non-empty subset of $\Omega(u, \alpha, \beta)$ for some $\alpha \geq 1$.

(i) If $\bar{K}(x) \equiv 1$ on $M$, then for any point $x \in M$ there exist a positive constant $r(x, \gamma)$ such that

$$
\frac{\log \operatorname{Vol}\left(B_{x}(r) \cap M(u, \gamma)\right)}{r^{2}} \geq\left(\frac{\beta \log 2}{2^{6} c_{*}}\right) \gamma^{\alpha} \text { for any } r \geq r(x, \gamma)
$$

where $c_{*}$ is a positive constant independent of $\alpha, \beta, \gamma$ and $x \in M$. In particular, it holds

$$
\liminf _{r \rightarrow+\infty} \frac{\log V_{x}(r)}{r^{2}}=+\infty \text { for any } x \in M
$$

(ii) If there exist a point $x_{1} \in M$, positive constants $c_{1}$ and $r_{*}$ such that $K(x) \geq c_{1} / d\left(x_{1}, x\right)$ for any $x \in M \backslash B_{x_{1}}\left(r_{*}\right)$ and $\gamma>\sup _{y \in B_{x_{1}}\left(r_{*}\right)} u(y)$, then there exists a positive constant $r\left(x_{1}, \gamma\right)$ such that

$$
\frac{\log \operatorname{Vol}\left(B_{x_{1}}(r) \cap M(u, \gamma)\right)}{r} \geq\left(\frac{\beta c_{1} \log 2}{2^{7} c_{*}}\right) \gamma^{\alpha} \text { for any } r \geq r\left(x_{1}, \gamma\right) \text {. }
$$

In particular, it holds

$$
\liminf _{r \rightarrow+\infty} \frac{\log V_{x_{1}}(r)}{r}=+\infty
$$

(iii) If there exist a point $x_{2} \in M$, positive constants $c_{2}$ and $r_{* *}$ such that $K(x) \geq c_{2} / d\left(x_{2}, x\right)^{2}$ for any $x \in M \backslash B_{x_{2}}\left(r_{* *}\right)$ and $\gamma>\sup _{y \in B_{x_{2}}\left(r_{* *}\right)} u(y)$, then there exists a positive constant $r\left(x_{2}, \gamma\right)$ such that

$$
\frac{\log \operatorname{Vol}\left(B_{x_{2}}(r) \cap M(u, \gamma)\right)}{\log r} \geq\left(\frac{\beta c_{2}}{2^{8} c_{*}}\right) \gamma^{\alpha} \text { for any } r \geq r\left(x_{2}, \gamma\right) \text {. }
$$


In particular, it holds

$$
\liminf _{r \rightarrow+\infty} \frac{\log V_{x_{2}}(r)}{\log r}=+\infty
$$

Remark 1.2. In Theorem 1.1 if $u^{*}:=\sup _{M} u<+\infty$, then we have only to consider an unbounded function $v:=1 /\left(u^{*}-u\right)$ such that $M\left(v, 1 /\left(u^{*}-\right.\right.$ $\gamma)$ ) is a non empty subset of $\Omega\left(v, 1, \beta \gamma^{\alpha+1}\right)$. The above volume growth properties follow immediately from this observation.

To show Theorem 1.1 we need the following lemma, which can be proved by a direct calculation.

LEMMA 1.3. Let $v$ (resp. $\omega$ ) be a smooth (resp. Lipschitz continuous) function on $M$ and let $\lambda$ be a smooth function on a real line $\mathbf{R}$. Then the following holds

$$
\begin{aligned}
& \operatorname{div}\left(\omega^{2} \operatorname{grad} \lambda\left(v^{2}\right)\right) \\
= & \omega^{2}\left\{2 \lambda^{\prime}\left(v^{2}\right)\left(v \Delta v+|d v|^{2}\right)+\lambda^{\prime \prime}\left(v^{2}\right)\left|d v^{2}\right|^{2}\right\}+4 \omega v \lambda^{\prime}\left(v^{2}\right)\langle d \omega, d v\rangle
\end{aligned}
$$

where $\Delta v=\operatorname{div}(\operatorname{grad} v)$.

Proof of Theorem 1.1. Since $M(u, \gamma)=M(u / \gamma, 1)$ and $\Omega(u, \alpha, \beta)=$ $\Omega\left(u / \gamma, \alpha, \beta \gamma^{\alpha}\right)$, we replace $u$ by $u / \gamma$ and set $\delta:=\beta \gamma^{\alpha}$. Hence we have $M(u, 1)(\neq \phi) \subset \Omega(u, \alpha, \delta)$. For any positive number $p>1$, setting $v^{p}=u^{p / 2}$ we obtain the following on the subset $M(u, 1)$ :

$$
v^{p}\left(\Delta v^{p}\right)+\left|d v^{p}\right|^{2} \geq\left(\frac{\delta p K}{2}\right) u^{p+\alpha}+\frac{2(p-1)}{p}\left|d v^{p}\right|^{2} .
$$

For a fixed positive number $\rho>1$ with $M(u, \rho) \neq \phi$, let $\lambda$ be a smooth function defined on real line such that $\lambda(t) \equiv 0$ if $t \leq 1, \lambda(t)>0, \lambda^{\prime}(t)>$ $0, \lambda^{\prime \prime}(t) \geq 0$ if $t>1$ and $\lambda(t) \equiv t$ if $t \geq \rho>1$. Since the Riemannian manifold $(M, g)$ is complete, for any fixed point $x \in M$ and $r>0$ there exists a Lipschitz continuous function $\omega_{r}$ with $0 \leq \omega_{r} \leq 1$ on $M$ such that $\omega_{r} \equiv 1$ on $B_{x}(r), \operatorname{supp} \omega_{r} \subset \overline{B_{x}(2 r)}$ and $\left|d \omega_{r}\right|^{2} \leq c_{*} / r^{2}$, where $c_{*}>0$ does not depend on $x$ and $r$. We apply Lemma 1.3 to $\omega=\omega_{r}^{p+\alpha}$ and $v=v^{p}$. Integrating the left hand side of Lemma 1.3 and using (1.4), we obtain for any $\varepsilon>0$ and $B_{x}(2 r, r):=B_{x}(2 r) \backslash B_{x}(r)$ the following:

$$
\frac{4(p-1)}{p} \int \omega_{r}^{2(p+\alpha)} \lambda^{\prime}\left(u^{p}\right)\left|d v^{p}\right|^{2}+\delta p \int K \omega_{r}^{2(p+\alpha)} \lambda^{\prime}\left(u^{p}\right) u^{p+\alpha}
$$




$$
\begin{aligned}
& \leq-4(p+\alpha) \int \omega_{r}^{2(p+\alpha)-1} \lambda^{\prime}\left(u^{p}\right) v^{p}\left\langle d \omega_{r}, d v^{p}\right\rangle \\
& \leq 2 \varepsilon \int \omega_{r}^{2(p+\alpha)} \lambda^{\prime}\left(u^{p}\right)\left|d v^{p}\right|^{2}+\frac{2(p+\alpha)^{2}}{\varepsilon} \int_{B_{x}(2 r, r)} \omega_{r}^{2(p+\alpha-1)} \lambda^{\prime}\left(u^{p}\right) u^{p}\left|d \omega_{r}\right|^{2}
\end{aligned}
$$

Taking $\varepsilon=(p-1) / p$ we obtain the following:

$$
\int K \omega_{r}^{2(p+\alpha)} \lambda^{\prime}\left(u^{p}\right) u^{p+\alpha} \leq \frac{2 c_{*}(p+\alpha)^{2}}{\delta(p-1) r^{2}} \int_{B_{x}(2 r, r)} \omega_{r}^{2(p+\alpha-1)} \lambda^{\prime}\left(u^{p}\right) u^{p} .
$$

On the other hand since $\alpha \geq 1$ and $\lambda^{\prime}\left(u^{p}\right)>0$ if and only if $u>1$, we obtain

$$
\begin{aligned}
& \int_{B_{x}(2 r, r)} \omega_{r}^{2(p+\alpha-1)} \lambda^{\prime}\left(u^{p}\right) u^{p} \\
& \leq\left(\int K \omega_{r}^{2(p+\alpha)} \lambda^{\prime}\left(u^{p}\right) u^{p(p+\alpha) /(p+\alpha-1)}\right)^{(p+\alpha-1) /(p+\alpha)} \\
& \times\left(\int_{B_{x}(2 r, r)} K^{-(p+\alpha-1)} \lambda^{\prime}\left(u^{p}\right)\right)^{1 /(p+\alpha)} \\
& \leq\left(\int K \omega_{r}^{2(p+\alpha)} \lambda^{\prime}\left(u^{p}\right) u^{p+\alpha}\right)^{(p+\alpha-1) /(p+\alpha)} \\
& \times\left(\int_{B_{x}(2 r, r)} K^{-(p+\alpha-1)} \lambda^{\prime}\left(u^{p}\right)\right)^{1 /(p+\alpha)}
\end{aligned}
$$

Hence we obtain the following:

$$
\begin{aligned}
& \int K \omega_{r}^{2(p+\alpha)} \lambda^{\prime}\left(u^{p}\right) u^{p+\alpha} \\
\leq & \left(\frac{2 c_{*}(p+\alpha)^{2}}{\delta(p-1) r^{2}}\right)^{p+\alpha} \int_{B_{x}(2 r, r)} K^{-(p+\alpha-1)} \lambda^{\prime}\left(u^{p}\right)
\end{aligned}
$$

for any $p>1$ and $r \geq r_{0}=r_{0}(x, \gamma)$ with $B_{x}\left(r_{0}\right) \cap M(u, 1) \neq \phi$. If $\inf _{y \in B_{x}(2 r, r)} K(y) \geq c_{\sigma} /(2 r)^{\sigma}$ for any $r>r_{0}$ and $c_{\sigma}>0$ with $\sigma \in\{0,1,2\}$, then setting $F(p, r):=\int_{B_{x}(r)} \lambda^{\prime}\left(u^{p}\right)$ and taking $p \geq \max \{2, \alpha\}$ we obtain the following from (1.5):

$$
F(p, r) \leq\left(\frac{2^{\sigma+4} c_{*} p}{\delta c_{\sigma} r^{2-\sigma}}\right)^{p+\alpha} F(p, 2 r)
$$


for any $r \geq r_{0}$.

We first show (i). For $\sigma=0$, put $c_{0}=1$ and set $p(r)=\delta r^{2} / 2^{5} c_{*} \geq$ $\max \{2, \alpha\}$ with $\delta=\beta \gamma^{\alpha}$. We define $F(r):=F(p(r), r)$. Then putting $\sigma=0$ in (1.6), we have the following:

$$
F(r) \leq\left(\frac{1}{2}\right)^{p(r)+\alpha} F(2 r)
$$

for any $r \geq r_{0}$. We fix $r$ such that $r \geq 2 r_{0}$. Since there exists $k \geq 1$ such that $2^{-(k+1)}<r_{0} / r \leq 2^{-k}$, by putting $r_{i}=2^{i} r_{0}$ and by (1.7) we obtain

$$
F\left(r_{0}\right) \leq\left(\frac{1}{2}\right)^{\sum_{i=0}^{k-1} p\left(r_{i}\right)+k \alpha} F\left(r_{k}\right) \leq\left(\frac{1}{2}\right)^{\delta r^{2} / 2^{6} c_{*}}\left(\frac{r_{1}}{r}\right)^{\alpha} F(r)
$$

for any $r \geq r_{1}$. Therefore there exists $r(x, \gamma)>0$ such that

$$
\frac{\log F(r)}{r^{2}} \geq\left(\frac{\beta \log 2}{2^{6} c_{*}}\right) \gamma^{\alpha}
$$

for any $r \geq r(x, \gamma)$. Since $u$ was replaced by $u / \gamma$ in the beginning and it may be assumed that $\sup _{\mathbf{R}} \lambda^{\prime}(t)=1$, it holds that $F(r) \leq \operatorname{Vol}\left(B_{x}(r) \cap M(u, \gamma)\right)$ for any $r \gg 0$. Therefore we can obtain the desired estimate in (i) from (1.8). To show (ii) (resp. (iii)) we have only to put $x=x_{1}\left(\operatorname{resp} . x=x_{2}\right.$ ), $\sigma=1$ (resp. $\sigma=2)$ in (1.6) and set $p(r)=\delta c_{1} r / 2^{6} c_{*}\left(\right.$ resp. $\left.p(r)=\delta c_{2} / 2^{7} c_{*}\right)$ in (1.7). The desired estimate follows from the same argument as above respectively. This completes the proof of Theorem 1.1 .

\section{§2. Maximum principle on a complete Riemannian manifold satisfying a certain volume growth condition}

Let $u$ be a smooth function on a Riemannian manifold $M$. For given constants $\alpha$ and $\beta$, we set

$$
\Gamma(u, \alpha, \beta):=\left\{x \in M ; u(x)>0 \text { and } \Delta u(x)<\beta u(x)^{\alpha+1}\right\} .
$$

First we show the following maximum principle:

THEOREM 2.1. Let $(M, g)$ be a complete Riemannian manifold of dimension $m \geq 1$. Suppose $M$ admits a point $x$ such that the following volume growth condition holds

$$
\liminf _{r \rightarrow+\infty} \frac{\log V_{x}(r)}{r^{2}}<+\infty
$$


For a given smooth function $u$ on $M$ such that $0<\sup _{M} u \leq+\infty$, and for $\alpha>0, \beta>0$, the following holds by setting $u^{*}=\sup _{M} u$ :

(i) $\Gamma(u, \alpha, \beta)$ is non-empty. In particular, $M$ admits no non-zero smooth solution $w \geq 0$ (resp. $v \geq 0$ ) satisfying $\Delta w \geq \beta w^{\alpha+1}$ (resp. $\Delta \log v \geq \beta v^{\alpha}$ ) on $M(c f .[\mathrm{Os}])$.

(ii) If $u^{*}<+\infty$ and $\sigma \geq-1$, then $\Gamma(u, \sigma, \beta)$ is non-empty. In particular, $M$ admits no non-zero smooth solution $v \geq 0$ with $\sup _{M} v<+\infty$ satisfying $\Delta v \geq \beta v^{\sigma+1}$ on $M$.

(iii) $u(x) \leq u^{*}(\alpha, \beta):=\sup _{y \in \Gamma(u, \alpha, \beta)} u(y)$ for any $x \in M$. Especially if $u^{*}(\alpha, \beta)$ is finite for a certain pair $(\alpha, \beta)$, then $u^{*}(\alpha, \beta)$ is independent of $\alpha$ and $\beta$ and hence $u^{*}=u^{*}(\alpha, \beta)$.

(iv) If $u^{*}<+\infty$ and $\Gamma(u):=\{x \in M ; u(x)>0$ and $\Delta u(x)<0\}$ is non-empty, then $u^{*}=\sup _{y \in \Gamma(u)} u$.

Proof for the case $\alpha \geq 1$. If $M$ is compact, then the above assertions are obvious. We may assume that $M$ is non-compact. First remark that the hypothesis $(*$ ) implies (i) by Theorem 1.1, (i). (ii) follows from Remark 1.2 and (i). To see (iii) if $u^{*}(\alpha, \beta)=+\infty$, then the assertion is trivial. If $u^{*}(\alpha, \beta)$ is finite and there exists a point $z \in M$ with $u^{*}(\alpha, \beta)<u(z)$, then it is clear that $M\left(u, u^{*}(\alpha, \beta)\right)$ is a non-empty subset of $\Omega(u, \alpha, \beta)$, which contradicts to the assumed growth condition $(*)$ by Theorem 1.1, (i). By the same argument, we can see the latter half of (iii). (iv) follows from (iii) immediately.

Proposition 2.2. Let $\left(X, g_{X}\right)$ be a complete Riemannian manifold of dimension $n \geq 1$ and let $f$ be a smooth function bounded from above on $X$. For any $\varepsilon>0$ take a point $y_{\varepsilon} \in X$ with $\sup _{X} f-\varepsilon^{2}<f\left(y_{\varepsilon}\right)$. Then there exists a point $x_{\varepsilon} \in X$ such that (i) $f\left(y_{\varepsilon}\right) \leq f\left(x_{\varepsilon}\right)$, (ii) $d_{X}\left(x_{\varepsilon}, y_{\varepsilon}\right) \leq \varepsilon$ and (iii) $|\operatorname{grad} f|\left(x_{\varepsilon}\right) \leq \varepsilon$, where $d_{X}$ is the distance function relative to $g_{X}$.

Proof. This was proved in $[\mathrm{H}]$ in the case $X=\mathbf{R}^{n}$. In this general case the proof is essentially the same and therefore omitted.

Now we are in a position to show the following our main result.

THEOREM 2.3. Under the volume growth condition (*) of Theorem 2.1 let $f$ be a smooth function bounded from above on $M$. Then for any given point $x \in M$ and $\varepsilon>0$ there exists a point $x_{\varepsilon} \in M$ depending on $x$ such that (i) $f(x) \leq f\left(x_{\varepsilon}\right)$, (ii) $|\operatorname{grad} f|\left(x_{\varepsilon}\right)<\varepsilon$ and (iii) $\Delta f\left(x_{\varepsilon}\right)<\varepsilon$. 
Proof. Since the assertion is trivial, if $f$ attains $f^{*}:=\sup _{M} f$, we suppose that $f$ does not attain $f^{*}$ on $M$. We put $\varepsilon_{*}:=\min \left\{\varepsilon, f^{*}-\right.$ $f(x)\} /\left(1+\min \left\{\varepsilon, f^{*}-f(x)\right\}\right)>0$. Set $w:=1 /\left(1+f^{*}-f\right)>0$ and $M_{p}:=M\left(w^{p}, 1-\varepsilon_{*}^{2}\right)$ for any positive integer $p$. Then clearly $M_{p} \subset M_{q}$ and $\partial M_{p} \cap \partial M_{q}=\phi$ if $p>q \geq 1$. On the other hand $\Gamma_{p}:=\Gamma\left(w^{p}, 1, \varepsilon_{*}\right)$ is non-empty by Theorem 2.1, (i). By the fact $(p-1) \Delta w^{p} \geq p w \Delta w^{p-1}$ for any $p \geq 2$ and $0<w<1$ on $M$, we obtain $\Gamma_{p} \subset \Gamma_{q}$ and $\partial \Gamma_{p} \cap \partial \Gamma_{q}=\phi$ if $p>q \geq 1$. Setting $\Sigma_{p}:=\Gamma_{p} \cap M_{p}, \Sigma_{p}$ is also non-empty and $\sup _{\Sigma_{p}} w^{p}=1$ by Theorem 2.1, (iii). In particular $\Sigma_{p} \subset \Sigma_{q}$ and $\partial \Sigma_{p} \cap \partial \Sigma_{q}=\phi$ if $p>q \geq 1$. Suppose $\Sigma_{p}$ converges to a non-empty subset $\Sigma_{\infty} \subset M$ containing a point $x_{\infty}$ as $p$ tends to infinity. Then $w$ should attain 1 at $x_{\infty}$. This is a contradiction. Hence $M \backslash \Sigma_{p}$ converges to the whole space $M$ as $p$ tends to infinity. This implies that $d_{M}\left(x_{*}, \Sigma_{p}\right)$ is unbounded for a fixed point $x_{*} \in M$. Set $\lambda_{p}:=\sup _{y \in \partial \Sigma_{p}} d_{M}\left(y, \partial \Sigma_{1}\right)$ for any $p>1$. Then $\lambda_{p} \in(0,+\infty]$ and $\lambda_{p}$ is non-decreasing in $p$. We claim that $\lim _{p \rightarrow+\infty} \lambda_{p}=+\infty$. Otherwise there exists a constant $c>0$ with $\lim _{p \rightarrow+\infty} \lambda_{p} \leq c<+\infty$. Then we obtain $\partial \Sigma_{p} \subset\left\{y \in M ; d_{M}\left(y, \partial \Sigma_{1}\right) \leq c\right\}$ for any $p \geq 1$. This implies $d_{M}\left(x_{*}, \Sigma_{p}\right) \leq d_{M}\left(x_{*}, \partial \Sigma_{1}\right)+c<+\infty$, which is a contradiction. Therefore there exists a large positive integer $p_{*}$ such that $\varepsilon_{*}<\lambda_{p} \leq+\infty$ for any integer $p$ with $p \geq p_{*}$. For a fixed $p \geq p_{*}$, there exists a point $y_{*} \in \partial \Sigma_{p}$ with $d_{M}\left(y_{*}, \partial \Sigma_{1}\right)>\varepsilon_{*}$. Clearly such a point admits a small positive constant $\delta_{*}$ such that $\overline{B_{z}\left(\varepsilon_{*}\right)} \subset \Sigma_{1}$ if $z \in B_{y_{*}}\left(\delta_{*}\right) \cap \Sigma_{p}$. Now we take a point $z_{\varepsilon} \in B_{y_{*}}\left(\delta_{*}\right) \cap \Sigma_{p}$. By Proposition 2.2, there exists a point $x_{\varepsilon} \in \overline{B_{z_{\varepsilon}}\left(\varepsilon_{*}\right)} \cap M_{p} \subset \Sigma_{1}$ such that $\left|d w^{p}\right|\left(x_{\varepsilon}\right) \leq \varepsilon_{*}$. If $p$ is large enough, then $x_{\varepsilon}$ is the desired point. This completes the proof of Theorem 2.3.

Using Theorem 2.3 we complete the proof of Theorem 2.1.

Proof of Theorem 2.1, (i) and (iii) for the case $0<\alpha<1$. To show (i) suppose $\Gamma(u, \alpha, \beta)$ is empty. Let $\lambda$ be a smooth function defined on the real line such that $\lambda(t)=0$ for $t \leq 0, \lambda^{\prime}(t)>0, \lambda^{\prime \prime}(t) \geq 0$ for $t \geq 0$ and $\lambda(t)=t$ for $t \geq c>0$. Taking $c$ arbitrarily we may assume that $v:=\lambda(u)$ satisfies $\Delta v \geq \beta v^{\alpha+1}$ on $\{v>\delta\} \neq \phi$ with $\delta:=\lambda(c)>0$. Set $w:=-1 /(1+v)^{p}$ with $p=\alpha / 2>0$ and $\varepsilon_{*}:=\min \left\{\sup _{M} w-w(\delta), 1\right\}>0$. By Theorem 2.3 for any $\varepsilon>0$ with $0<\varepsilon<\varepsilon_{*}$, there exists a point $x_{\varepsilon} \in M$ such that (1) $\sup _{M} w-\varepsilon<w\left(x_{\varepsilon}\right)$, (2) $|\operatorname{grad} w|\left(x_{\varepsilon}\right)<\varepsilon$, (3) $\Delta w\left(x_{\varepsilon}\right)<\varepsilon$. Since $\Delta w=p \Delta v /(1+v)^{p+1}-p(p+1)|\operatorname{grad} v|^{2} /(1+v)^{p+2}$ 
and $\Delta v\left(x_{\varepsilon}\right) \geq \beta v^{\alpha+1}\left(x_{\varepsilon}\right)$, we get the following :

$$
\left(\frac{v\left(x_{\varepsilon}\right)}{1+v\left(x_{\varepsilon}\right)}\right)^{2 p+1} \beta \leq\left(\frac{2 p+1}{p^{2}}\right) \varepsilon .
$$

This implies $v^{*}:=\sup _{M} v<+\infty$ and so there exists $C>0$ independent of $\varepsilon$ such that $v\left(x_{\varepsilon}\right)^{2 p+1} \leq C \varepsilon$. Letting $\varepsilon \rightarrow 0$ we obtain $v^{*}=0$, which implies $u \equiv 0$ on $\{u>0\} \neq \phi$. This is a contradiction. To show (iii) suppose $u^{*}(\alpha, \beta)$ is finite and there exists a point $z \in M$ with $u^{*}(\alpha, \beta)<$ $u(z)$. Then $u$ satisfies that $\Delta u \geq \beta u^{\alpha+1}$ on $\left\{u>u^{*}(\alpha, \beta)\right\} \neq \phi$. By the similar argument as in the proof of (i) we can conclude $u \equiv u^{*}(\alpha, \beta)$ on $\left\{u>u^{*}(\alpha, \beta)\right\} \neq \phi$, which is a contradiction. This completes the proof of Theorem 2.1.

Remark 2.4. For a given smooth monotone increasing function $h(r)$ satisfying $c_{1}=\int_{1}^{+\infty} d r / r h(r)<+\infty$, the example stated in the introduction is constructed as follows: Take a function $f(r)$ such that $(1) f(0)=0$, $f^{\prime}(0)=1, f(r)>0, f^{\prime}(r)>0, f^{\prime \prime}(r) \geq 0$ if $r>0$, and $(2) f(r)=$ $c_{2}\left(\exp \left(r^{2} h(r)\right)\right)^{\prime}$ with $c_{2}>0$ (is determined later) if $r \geq 1$. We define a complete metric $g$ on $\left([0,+\infty) \times S^{1},(r, \theta)\right)$ by $g=d r^{2}+f(r)^{2} d \theta^{2}$. Since $\Delta u(r)=\left(f(r) u_{r}(r)\right)_{r} / f(r), \Delta u \equiv 1$ and $\sup u \leq c_{1}$ if $u(r):=$ $\int_{0}^{r}\left\{\left(\int_{0}^{t} f(s) d s / f(t)\right\} d t\right.$. Setting $v(r)=$ the volume of $S^{1}=S^{1} \times\{r\}$ relative to $g, \quad(\log (f(r) / v(r)))_{r} \equiv 0$ for any $r>0$ (cf. [G-W]). Put $c_{2} \equiv$ $f(r) / v(r)>0$. Since $V_{0}(r)=\int_{0}^{r} v(t) d t$, setting $h_{0}(r)=\left(\log V_{0}(r)\right) / r^{2}$, it can be easily verified that $h(r) / 2 \leq h_{0}(r) \leq 2 h(r)$ for any $r \gg 0$.

\section{$\S 3$. Applications}

The maximum principle is closely related to several problems in differential geometry and geometric function theory; i.e., Liouville type theorem and Ahlfors-Schwarz type lemma for maps, minimal submanifolds in $\mathbf{R}^{n}$, conformal deformation of Riemann structure, etc. By replacing curvature condition by volume growth condition to assure the maximum principle, a few results related to those problems can be stated without proof. With respect to the details of the proof and the related topics the reader should see the references cited in the end of each statement.

THEOREM 3.1. Let $(M, g)$ be a complete Riemannian manifold of dimension $m \geq 1$. Suppose $(M, g)$ admits a point $x_{1}$ (resp. $\left.x_{2}\right)$ such that

$$
\liminf _{r \rightarrow+\infty} \frac{\log V_{x_{1}}(r)}{r}<+\infty \quad\left(\text { resp. } \quad \liminf _{r \rightarrow+\infty} \frac{\log V_{x_{2}}(r)}{\log r}<+\infty\right)
$$


Then for a non-negative continuous function $K_{1}\left(\right.$ resp. $\left.K_{2}\right)$ on $M$ satisfying $K_{1}(x) \geq c_{*} / d\left(x_{1}, x\right), x \in M \backslash B_{x_{1}}\left(r_{*}\right)$ (resp. $K_{2}(x) \geq c_{*} / d\left(x_{2}, x\right)^{2}, x \in$ $\left.M \backslash B_{x_{2}}\left(r_{*}\right)\right)$ for $r_{*} \gg 0$ and $c_{*}>0$, there exists no non-zero smooth solution $u \geq 0$ satisfying $\Delta u \geq K_{1} u^{\sigma}$ (resp. $\Delta u \geq K_{2} u^{\sigma}$ ) on $M$ if $\sigma \geq 2$. In particular, there exists no non-zero smooth solution $u \geq 0$ with $\sup _{M} u<$ $+\infty$ satisfying $\Delta u \geq K_{1} u^{\sigma}$ (resp. $\Delta u \geq K_{2} u^{\sigma}$ ) on $M$ if $\sigma \geq 0$. Furthermore there exists no non-zero smooth solution $v \geq 0$ satisfying $\Delta \log v \geq K_{1} v$ (resp. $\Delta \log v \geq K_{2} v$ ) on $M$ (cf. [C-L], Theorems 2.1 and 5.1, [L], [N], §3, [R-R-S], Theorems 3.1, 4.1 and 4.2, and [Y-2], Theorems 1 and 5).

Remark 3.2. In Theorem 3.1 if $m \geq 3$, then the non-negativity condition of $K_{1}$ (resp. $K_{2}$ ) can be weakened in the following way: Under the hypothesis of Theorem 3.1 there exists a constant $\varepsilon>0$ such that there is no positive smooth solution satisfying $\Delta u \geq K_{1} u^{\sigma}$ (resp. $\Delta u \geq K_{2} u^{\sigma}$ ) provided that $\sigma \geq 2$ and $K_{1} \geq-\varepsilon$ (resp. $K_{2} \geq-\varepsilon$ ) on $M$ (cf. $\S 1$, Theorem 1.1 and [R-R-S], Proposition 3.5).

THEOREM 3.3. Under the volume growth condition $(*)$ of Theorem 2.1 the following holds :

(i) Suppose $h:(M, g) \rightarrow\left(N, g_{N}\right)$ is an isometric immersion into an Hadamard manifold $\left(N, g_{N}\right)$ of dimension $n>m$ such that the mean curvature $H$ of $h$ satisfies $H_{*}:=\sup _{M}|H|<+\infty$, and $h(M) \subset B_{y_{*}}(R), y_{*} \in N$. Then $R \geq \frac{1}{H_{*}}$ (cf. [Kr], Theorem 3.1).

(ii) Suppose $f: M \rightarrow \mathbf{R}^{n}$ is a smooth map whose energy density $e(f)$ satisfies $e(f) \geq c>0$ on $M$, and there exists a unit vector $\xi$ at the origin of $\mathbf{R}^{n}$ such that $\langle\xi, f(x) /\|f(x)\|\rangle \geq \delta>0$ for any $x \in M$; i.e., $f(M)$ lies inside of a non-degenerate cone of $\mathbf{R}^{n}$. Then there exists a point $y \in M$ such that the tension field $\tau(f)$ of $f$ does not vanish at $y(c f .[\mathrm{B}-\mathrm{K}]$, Theorem 3 and $[\mathrm{Om}]$, Theorem $\mathrm{B})$.

COROLLARY 3.4. Under the volume growth condition (*) of Theorem 2.1, $M$ can not be isometrically immersed as a minimal submanifold into a bounded set of a Riemannian manifold admitting a smooth strictly convex function. In particular, if $f: M \rightarrow \mathbf{R}^{n}$ is a minimal isometric immersion; then $f(M)$ does not lie inside of any non-degenerate cone of $\mathbf{R}^{n}(c f .[\mathrm{Ks}]$, Proposition 2 and Remarks).

THEOREM 3.5. Let $\left(N, g_{N}\right)$ be a Riemannian manifold with sectional curvature bounded above by a constant $K$ and let $B_{R}\left(y_{*}\right)$ be inside the cut 
locus of $y_{*} \in N(R<\pi / 2 \sqrt{K}$ if $K>0)$. Under the volume growth condition (*) of Theorem 2.1 suppose $h: M \rightarrow N$ is a smooth map with $|\tau(h)| \leq$ $\tau_{*}, \tau_{*} \in[0,+\infty)$, and $h(M) \subset B_{R}\left(y_{*}\right)$. Then setting $e_{*}:=\inf _{M} e(h)$ for the energy density $e(h)$ of $h$, the following holds (cf. [C-X], Theorem 3.1 and [R-R], Theorem 2.17) :

$$
\begin{array}{lll}
R \geq \sqrt{K} \tan ^{-1}\left(2 \sqrt{K} e_{*} / \tau_{*}\right) & \text { when } K>0 \\
R \geq 2 e_{*} / \tau_{*} & \text { when } K=0 \\
R \geq \sqrt{-K} \tanh ^{-1}\left(2 \sqrt{-K} e_{*} / \tau_{*}\right) & \text { when } K<0
\end{array}
$$

COROLLARY 3.6. Under the volume growth condition (*) of Theorem 2.1 , suppose $M$ is a submanifold in $\mathbf{R}^{n}$ with parallel mean curvature and the image of $M$ of the Gauss map $\mathcal{G}: M \rightarrow \mathbf{G}_{m, n-m}$ lies in the geodesic ball $B_{R}\left(y_{*}\right) \subset \mathbf{G}_{m, n-m}, \quad R<\pi / 2 \sqrt{K}$ and $y_{*} \in \mathbf{G}_{m, n-m}, \quad\left(K=1\right.$ if $\mathbf{G}_{m, n-m}$ is the sphere, otherwise $K=2$ ), then $M$ should be minimal (cf. [C-X], Theorem 3.3, [H-J-K], §4, Theorems 5 and 7, and [R-R], Corollary 2.24).

THEOREM 3.7. Let $\left(M, \omega_{M}\right)$ be a complete Hermitian manifold of dimension $m$ whose scalar curvature bounded from below by $-K_{1}$ and let $N$ be a complex manifold of the same dimension with a volume form $V_{N}$ whose Ricci form is negative definite and satisfies $\wedge^{m}(\sqrt{-1} / 2) \partial \bar{\partial} \log V_{N} \geq K_{2} V_{N}$. Suppose $\left(M, \omega_{M}\right)$ satisfies the volume growth condition $(*)$ of Theorem 2.1 and $f: M \rightarrow N$ is a holomorphic map whose Jacobian does not vanish at some point. Then $K_{1}>0$ and $\sup f^{*} V_{N} / V_{M} \leq K_{1}^{m} / m^{m} K_{2}$, where $V_{M}$ is the volume form relative to $\omega_{M}$ (cf. [M-Y], $\left.1,[\mathrm{Ry}],[\mathrm{Y}-3]\right)$.

\section{REFERENCES}

[B-K] C. H. Baikoussis and T. H. Koufogiorgos, Harmonic maps into a cone, Arch. Math., 40 (1983), 372-376.

[C-L] K.-S. Cheng and J.-T. Lin,, On the elliptic equations $\Delta u=K(x) u^{\sigma}$ and $\Delta u=K(x) e^{2 u}$, Trans. Amer. Math. Soc., 304 (1987), 639-668.

[C-Y] S. Y. Cheng and S. T. Yau, Differential equations on Riemannian manifolds and their geometric applications, Comm. Pure Appl. Math., 28 (1975), 333-354.

[C-X] Q. Chen and Y. L. Xin, A generalized maximum principle and its applications in geometry, Amer. J. Math., 114 (1992), 355-366.

[G-W] R. E. Greene and Y. Wu, Function theory on manifolds which possess a pole, Lecture Notes in Math., 699, Springer-Verlag. 
[H] J. B. Hiriart-Urruty, A short proof of the variational principle for approximate solutions of a minimaization problem, Amer. Math. Monthly, 90 (1983), 206-207.

[H-J-W] J. Hildebrandt, J. Jost and K. O Widman, Harmonic mappings and minimal submanifolds, Invent. Math., 62 (1980), 269-298.

[Kr] L. Karp, Differential inequalities on complete Riemannian manifolds and applications, Math. Ann., 272 (1985), 449-459.

[Ks] A. Kasue, Estimates for solutions of Poisson equations and their applications to submanifolds, Lecture Notes in Math. Differential Geometry of submanifolds, 1090, Springer-Verlag (1984), pp. 1-14.

[L] P. Li, Uniqueness of $L^{1}$ solutions for the Laplace equation and the heat equation on Riemannian manifolds, J. Differential Geom., 20 (1984), 447-457.

[L-S] P. Li and R. Schoen, $L^{p}$ and mean value properties of subharmonic functions on Riemannian manifolds, Acta Math., 153 (1984), 279-301.

[M-Y] N. Mok and S. T. Yau, Completeness of the Kähler-Einstein metric on bounded domains and the characterization of domains of holomorphy by curvature conditions, Proc. Sympos. Pure Math., 39, Amer. Math. Soc., Providence, R. I. (1983), pp. 41-59.

[N] W.-M. Ni, On the elliptic equation $\Delta u+K(x) e^{2 u}=0$ and conformal metrics with prescribed Gaussian curvature, Invent. Math., 66 (1982), 343-352.

[Om] H. Omori, Isometric immersions of Riemannian manifolds, J. Math. Soc. Japan, 19 (1967), 205-214.

[Os] R. Osserman, On the inequality $\Delta u \geq f(u)$, Pacific J. Math., 7 (1957), 1641-1647.

[R-R] . A. Ratto and M. Rigoli, Elliptic differential inequalities with applications to harmonic maps, J. Math. Soc. Japan, 45 (1993), 321-337.

[R-R-S] A. Ratto, M. Rigoli and G. Setti, On the Omori-Yau maximum principle and its applications to differential equations and geometry, J. Funct. Anal., 134 (1995), 486-510.

[Ry] H. L. Royden, The Ahlfors-Schwarz lemma in several complex variables, Comment. Math. Helv., 55 (1980), 547-558.

[Y-1] S. T. Yau, Harmonic function on complete Riemannian manifolds, Comm. Pure Appl. Math., 28 (1975), 201-228.

[Y-2] Some function theoretic properties of complete Riemannian manifolds and their applications to geometry, Indiana Univ. Math. J., 25 (1976), 656-670.

[Y-3] - A general Schwarz lemma for Kähler manifolds, Amer. J. Math., 100 (1978), 197-203.

Department of Mathematics

Graduate School of Science

Osaka University

Toyonaka, Osaka, 560-0043

Japan

kensho@math.wani.osaka-u.ac.jp 